\title{
Circulating RP11-445H22.4 and TINCR are potential biomarkers for invasive ductal carcinoma of the breast
}

Short running title: Circulating lncRNAs in breast carcinoma

\author{
Mohammad-Taher Moradi a ${ }^{\text {, Zohreh Rahimi }}{ }^{\text {b, * }}$
}

${ }^{a}$ Student Research Committee, Kermanshah University of Medical Sciences, Kermanshah, IRAN

b Medical Biology Research Center, Health Technology Institute, Kermanshah University of Medical Sciences, Kermanshah, IRAN

*Corresponding author at: Medical Biology Research Center, Medical School, Kermanshah University of Medical Sciences, Parastar Avenue, P.O. Box 6714869914, Kermanshah, Iran.

Email addresses: zrahimi@kums.ac.ir; rahimizus@yahoo.com 


\begin{abstract}
Introduction. Recently introduced molecules namely long non-coding RNAs (lncRNAs) are associated with various diseases, including breast cancer, and they may have the potential to act as biomarkers. Invasive ductal carcinoma (IDC) is a frequently observed type of breast cancer that diagnosis through immunohistochemical methods. However, no reliable non-invasive markers such as circulating markers have been introduced for it, so far.
\end{abstract}

Methods. The relative quantitation (RQ) of two lncRNAs, RP11-445H22.4 and TINCR, was measured in tumoral tissues and paired adjacent non-tumoral tissues (PANTs) as well as in plasma samples using real-time polymerase chain reaction (RT-PCR). Furthermore, promoter methylation of the two lncRNAs genes was assessed using methylation-specific PCR (MSP). In order to compare the diagnostic values, plasma levels of CA 15-3 were measured as a conventional circulating biomarker using ELISA. The receiver operating characteristic (ROC) curve was used to illustrate the sensitivity and specificity of the potential biomarkers. Results. The RP11-445H22.4 and TINCR genes were upregulated in tumor tissues compared to PANTs $\left(\mathrm{RQ}_{\mathrm{RP} 11-445 \mathrm{H} 22.4}=2.7, \mathrm{RQ}_{\mathrm{TINCR}}=4.4\right)$. Furthermore, the presence of $\mathrm{RP} 11-445 \mathrm{H} 22.4$ and $\mathrm{TINCR}$ in the plasma of patients was significantly more than healthy controls $\left(\mathrm{RQ}_{\mathrm{c} \cdot \mathrm{RP} 11-445 \mathrm{H} 22.4}=4.12\right.$, $\left.\mathrm{RQ}_{\mathrm{c} . \mathrm{TINCR}}=4.16, \mathrm{P}<0.001\right)$. The promoters of RP11-445H22.4 and TINCR genes were hypomethylated in the tumoral tissues with a negative correlation with their gene expression. Conclusion. Based on the findings, it can be speculated that the expression levels of these two lncRNAs as well as their promoter methylation can be considered as a therapeutic target and potential biomarker for breast carcinoma.

Keywords: Biomarker; Breast cancer; RP11-445H22.4; IncRNA; TINCR 


\section{Introduction}

Breast cancer is one of the primary health concerns worldwide [1]. According to the report published by the Non-Communicable Diseases Research Center (NCDRC) of Iran, breast cancer is the most prevalent type of cancer among women in Kermanshah in western Iran (33.02 new cases per 100000 women in 2016) [2]. Nowadays, it has been well demonstrated that the earlier diagnosis of breast cancer is associated with the better the survival rate will be. Conventional serum biomarkers for the detection of breast cancer are not highly convincing for early detection. In recent years, new types of potential biomarkers have been introduced for the prognosis, diagnosis, and treatment of cancer, involving microRNAs and long noncoding RNAs (lncRNAs) [3, 4]. LncRNAs are classified as transcripts with more than 200 nucleotides, with important functions in biological processes. [5]. Abnormal promoter methylation takes part in tumorigenesis, including in human breast carcinoma [6]. Therefore, IncRNA-associated methylation received increased attention, for example, the investigation of the role of lncRNA MEG3 in breast cancer [7, 8]. Hence, the identification of new genes and mechanisms for their regulation and functions may result in therapeutic approaches or novel biomarkers for breast carcinoma.

KCNK15 and WISP2 antisense RNA 1 (KCNK15-AS1) a.k.a. RP11-445H22.4, is a newly identified antisense lncRNA with a length of 862 nucleotides, which has been found in the reverse strand of human chromosome 20q13.12 [9]. Tissue differentiation-inducing long noncoding RNA (TINCR) is a lncRNA which is necessary for regular epidermal differentiation [10]. The TINCR gene is located on human chromosome 19 and produces a 3.7-kb RNA transcript [11]. 
The main objective of the current study was to assess tissue and plasma expression of the RP11445H22.4 and TINCR in a sample of female Kurdish patients with invasive ductal carcinoma (IDC) of the breast. Moreover, to our knowledge, this is the first study to evaluate the promoter methylation patterns of these two lncRNAs.

\section{Methods}

2.1. Participants and sample collection. Patients were recruited at the time of the diagnosis at the Bistoon Hospital, between 2016 and 2017. The ethical committee of Kermanshah University of Medical Sciences approved this study, and informed consent was signed by all subjects. Only new confirmed patients with invasive ductal carcinoma of breast were entered in the study without previously diagnosed any malignancy and without manifestation of acute diseases or injury at the time of sampling. The status of hormone receptors and other clinicopathological data were extracted from the patient's medical history. The samples consisted of 20 paired frozen tumoral tissues and adjacent nontumoral tissues (PANTs) from patients with IDC of the breast. Moreover, 40 patients who were sampled only for taken peripheral blood at the time of diagnosis were studied. In addition, the blood sample was taken from 40 healthy volunteers. The results of histopathological examination confirmed by an expert pathologist in all patient samples to be invasive ductal carcinoma and paired adjacent margin tissues were confirmed as free of cancer cells.

2.2. Stock of plasma samples. The whole peripheral blood samples were collected in $5 \mathrm{ml}$ EDTA containing tubes. To separate cellular fraction from plasma, samples centrifuged 
with $3500 \mathrm{~g}$ for $20 \mathrm{~min}$ around one hour after blood collection. The collected plasma was stored at $-80{ }^{\circ} \mathrm{C}$.

2.3. Quantitative real-time PCR ( $q R T-P C R)$. In order to extract RNA, fresh frozen tissues were powdered in liquid nitrogen, afterward, total RNA was isolated by Qiazol reagent (QIAGEN, US) according to the manufacturer's procedure. In addition, total RNA was isolated from 200 microliters of obtained plasma by the miRNeasy Plasma/ Serum extraction Kit (QIAGEN, US) and was eluted into $14 \mu \mathrm{l}$ of elution buffer. The concentration and purity of the extracted RNA were assessed by the Nanodrop spectrophotometer (Thermo Scientific, Waltham, MA). Complementary DNA (cDNA) was synthesized by applying a QuantiTect Reverse Transcription Kit (QIAGEN, US) from the isolated total RNA.

The qRT-PCR assay was performed by specific primers for RP11-445H22.4, TINCR, and U6 snRNA, as an internal control, using by the Rotor-gene (Corbett Research, Germany) with SYBR Premix Ex Taq II (TaKaRa, China). The PCR reaction conditions were an initial denaturation at $95^{\circ} \mathrm{C}$ for $30 \mathrm{sec}$, followed by $45 \mathrm{PCR}$ cycles at $95{ }^{\circ} \mathrm{C}$ for $10 \mathrm{sec}$ and $60{ }^{\circ} \mathrm{C}$ for $60 \mathrm{sec}$. We used the $2^{-} \Delta \Delta^{\mathrm{Ct}}$ method to determine the relative quantitation (RQ) in the cancerous samples relative to the control samples.

2.4. Methylation Specific PCR (MSP). DNA was extracted from frozen tissue using previously described modified salting-out DNA extraction method [12]. In order to Bisulfite treatment of extracted DNA was performed as follow, $30 \mu \mathrm{L}$ of melted $2 \%$ low melt agarose was added to about $200 \mathrm{ng}$ DNA and was incubated at $65{ }^{\circ} \mathrm{C}$ for a few minutes. Then for denaturation of DNA strands, it was incubated with $\mathrm{NaOH}$ at $80{ }^{\circ} \mathrm{C}$ for 
15 minutes and resolidify the agarose bead by chilling on ice. Treatment was done by adding $10 \mathrm{mM}$ hydroquinone and $40.5 \%$ Sodium bisulfite at $50{ }^{\circ} \mathrm{C}$ for about 4 hours. Finally, in order to stop bisulfite reaction and desulfonation, the beads were washed with TE buffer, 0.2 M NaOH, 1M hydrochloric acid, and pure water, respectively. The washed beads were diluted in $30 \mathrm{ul}$ deionized water, heated at $80{ }^{\circ} \mathrm{C}$ and aliquoted for PCR reaction.

An MSP method was used in the determination of the methylation status of the two lncRNAs gene promoters. For this reason, the promoters of these genes and CpG islands were has predicted by the UCSC Genome Browser (https://genome.ucsc.edu/) on Human (GRCH38/hg38) assembly and confirmed by the Genomatix software (https://www.genomatix.de/). The MSP primers for RP11-445H22.4 and TINCR genes were designed using the MethPrimer (https://www.urogene.org/methprimer/). The MSP-PCR was performed using two pairs of specific primers for each gene (Table 1); one pair specific for methylated and the other for the unmethylated template.

To prepare $25 \mu \mathrm{l}$ MSP reaction, $5 \mu \mathrm{l}$ bisulfite-treated DNA, $1 \mu \mathrm{l}$ of each forward and reverse primer $(10 \mu \mathrm{M}), 12 \mu \mathrm{l}$ PCR MasterMix (Amplicon), and $6 \mu 1 \mathrm{ddH} 2 \mathrm{O}$ were mixed. MSP conditions included an initial denaturation at $95^{\circ} \mathrm{C}$ for $2 \mathrm{~min}$, followed by 32 cycles of $30 \mathrm{sec}$ at $94^{\circ} \mathrm{C}$, annealing at $58^{\circ} \mathrm{C}$ for $30 \mathrm{sec}$, and extension at $72^{\circ} \mathrm{C}$ for $30 \mathrm{sec}$, with a final extension at $72^{\circ} \mathrm{C}$ for $5 \mathrm{~min}$. To perform quality control, we used the EpiTect PCR Control DNA Set (Qiagen, Germany), and also each reaction repeated, at least, two times.

2.5. Stability analysis of c.RP11-445H22.4 and c.TINCR in plasma. In order to the assessment of c.RP11-445H22.4 and c.TINCR stability in plasma, three plasma samples were examined; each 
sample divided into four equal parts and incubated at room temperature for 0-, 6-, 12-, and 24hours.

2.6. Measurement of CA 15-3 in plasma. The CA15-3 test was done in accordance with the instructions of the manufacturer. The plasma level of CA15-3 was measured in 40 patients with IDC and 40 healthy controls using the ELISA kit (Monobind, USA). The company recommended the threshold value equal to $35 \mathrm{UI} / \mathrm{mL}$. The samples were considered to be positive when its level exceeded a threshold value; if not, they were considered to be negative.

2.7. Statistical Analysis Receiver operating characteristic (ROC) curves and the area under the ROC curve (AUC) were applied to calculate the feasibility of circulating lncRNAs application as a diagnostic tool. Comparisons between two and three groups were analyzed using Student's ttest and One-Way ANOVA, respectively. A paired t-test was used for comparison of lncRNAs expression between tumor tissue and paired adjacent non-tumor tissues. Statistical analyses were done by SPSS (v.16.0) software. A P-value of less than 0.05 was considered significant.

\section{Results}

All participants were women with Kurdish ethnic background from Kermanshah Province of Iran. The mean age of patients was about 49 years (range of 30-68), while the healthy controls had a similar age distribution with a mean of 48 years (range of $26-64, p=0.14)$. A number of important clinicopathological characteristics, as well as the results of the immunohistochemical (IHC) analyses of the patients, are presented in Table 2. 
3.1. The expression of the RP11-445H22.4 and TINCR in tumoral tissues. Using qRT-PCR, the relative expression of the two lncRNAs were measured in 20 pairs of breast tumor tissues and PANTs samples. The expression levels of RP11-445H22.4 and TINCR were significantly higher in tumor tissues compared to PANT tissues $\left(\mathrm{RQ}_{\mathrm{RP} 11-445 \mathrm{H} 22.4}=2.7, \mathrm{RQ}_{\mathrm{TINCR}}=4.4\right.$, Figure $\left.1 \mathrm{~A}\right)$. Given that the lncRNAs in circulation were most probably originated from the tumor [13], we only evaluated the presence of RP11-445H22.4 and TINCR in the plasma since they had been overexpressed in tumor tissues.

3.2. The promoters of RP11-445H22.4 and TINCR were hypomethylated. The current study was designed to determine whether gene expressions of the above mentioned lncRNAs are influenced by promoter DNA methylation. The promoter of $R P 11-445 H 22.4$ gene was un-methylated in 18 (90\%) and hemi-methylated in $2(10 \%)$ tumoral tissues, while it was fully-methylated in 12 $(60 \%)$ and hemi-methylated in $8(40 \%)$ paired adjacent non-tumoral tissues. Also, the promoter of TINCR was un-methylated in $16(80 \%)$ and hemi-methylated in $4(20 \%)$ tumoral tissues, while it was fully-methylated in $15(75 \%)$ and hemi-methylated in $5(25 \%)$ of PANT tissues. Overall, we found that the promoters of $R P 11-445 H 22.4$ and TINCR were more unmethylated in the breast tumoral tissues, while they were more methylated in the PANT tissues $(\mathrm{P}<0.001$; Figure 2). Pearson's correlation analysis demonstrated that the tissue expression levels of each of the two lncRNAs were negatively correlated with their methylation status $\left(\mathbf{r}_{\mathrm{RP} 11-445 \mathrm{H} 22.4}=-0.68\right.$, $95 \%$ CI (-0/82 to $-0 / 46), \mathbf{r}_{\mathrm{TINCR}}=-0.84,95 \% \mathrm{CI}(-0 / 91$ to $-0 / 72)$.

3.3. Significant elevation of plasma levels of c.RP11-445H22.4 and c.TINCR in patients. The plasma levels of c.RP11-445H22.4 and c.TINCR were significantly higher in IDC patients compared to healthy donors (the mean RQ ${ }_{\text {c.RP11-445H22.4 }}=4.12$ and $\mathrm{RQ}$ c.TINCR $=4.16$ ). In fact, the 
level of both circulating lncRNAs in IDC of breast increased more than four fold in breast cancer patients $(\mathrm{P}<0.0001$, Figure 1b). The analysis showed no significant correlation between RP11445H22.4 and TINCR expression and the status of Hormone-Responsiveness Markers (i.e., ER, PR, or HER2) or tumor stage.

3.4. Diagnostic Value of c.RP11-445H22.4 and c.TINCR in the plasma of patients. In order to evaluate the potential clinical application of the circulating lncRNAs, the ROC curve was constructed using the data of 80 IDC patients and cancer-free controls, followed by comparison to a conventional plasma biomarker, i.e., cancer antigen 15-3 (CA 15-3) (Figure 3). The AUCs (area under curve) of c.RP11-445H22.4 and c.TINCR for differentiating patients from controls were 0.94 (95\% CI: 0.88 to $0.99, P<0.001)$ and 0.86 (95\% CI: 0.79 to $0.94, P<0.001)$, respectively (Figure 3). The optimal sensitivity and specificity were calculated for c.RP11$445 \mathrm{H} 22.4$ was $95 \%$ and $80 \%$, respectively, and for c.TINCR was $80 \%$ and $72.5 \%$, respectively. Moreover, in order to compare the diagnostic value of c.RP11-445H22.4 and c.TINCR with that of a conventional circulating biomarker, CA15-3 was measured in the plasma samples of the patients and cancer-free donors. Sensitivity and specificity for CA15-3 were $87 \%$ and $77 \%$, respectively $(\mathrm{AUC}=0.87,95 \% \mathrm{CI}: 0.78-0.95, P<0.001)$.

3.5. Stability analysis of c.RP11-445H22.4 and c.TINCR in plasma. In order to assess the stability of c.RP11-445H22.4 and c.TINCR in plasma, three plasma samples were examined; each sample was divided into four equal parts and incubated at room temperature for $0,6,12$, and 24 hours, respectively. Since no considerable variation was observed in plasma levels of c.RP11-445H22.4 and c.TINCR $(P<0.001$, Figure 4), the results indicated that they were stable in plasma. 


\section{Discussion}

According to the definition provided by World Health Organization (WHO), "a biomarker is any substance, structure, or process that can be measured in the body or its products and can influence or predict the incidence of outcome or disease". A cancer biomarker evaluates the risk of cancer development, progression, and recurrence [14, 15]. CA15-3, CEA, CA125, and CA19-9 are conventional blood-based biomarkers for cancer [16]. Since these factors are not specific and have low sensitivity, attempts to discover new biomarkers seem necessary.

It has been demonstrated that long non-coding RNAs (IncRNAs) are dysregulated in tumors, and they can show a high degree of tissue and disease-specificity [17]. Since IncRNAs have been detected within cells and body fluids of patients, such as serum, plasma, and urine, it has been speculated that they could be used as potential diagnostic and prognostic biomarkers for different types of cancer [17]. A known example of lncRNAs, with approval from the US Food and Drug Administration (FDA), is Prostate Cancer Antigen 3 (PCA3, a prostate-specific lncRNA) [18, 19]. Nowadays, the urinary PCA3 is used broadly, and it is even preferable to digital rectal examination and the serum prostate-specific antigen (PSA) test [17].

In this study, the increased expression of RP11-445H22.4 was detected in breast tumor tissues, as well as in the plasma of patients. Comparing the sensitivity of circulating RP11-445H22.4 with CA15-3 in the plasma of IDC patients revealed the potential diagnostic value of this antisense lncRNA. A review of the literature shows that there are a limited number of studies regarding RP11-445H22.4. Two separate studies on the Chinese population reported high expression of circulating $R P 11-445 H 22.4$ in breast cancer patients $[9,20]$. However, two other 
studies reported the downregulation of $R P 11-445 H 22.4$ in the tissue of pancreatic and gastric cancer patients $[21,22]$.

Deacetylation of histones and methylation of DNA are two epigenetic mechanisms that regulate gene expression [22]. One of the most important outcomes of hypomethylation is the overexpression of proto-oncogenes, which ultimately promotes cancer [23]. Most recently, it was reported that the promoter of $R P 11-445 H 22.4$ is methylated in gastric tumors, especially in stages III and IV [22]. However, no study has been reported on promoter methylation of this gene in breast tumors. For the first time, we reported a low-frequency rate of methylation in the promoter of the RP11-445H22.4 gene in breast cancer tissues.

TINCR is a lncRNA with an oncogenic role in breast carcinoma $[10,24]$. It has been uncovered that TINCR is regulated by SP1, miR-137/miR-133a, and CREB-binding protein (CBP)mediated H3K27 acetylation [10, 25, 26]. Acetylation of H3K27 by CBP in the promoter region of TINCR is involved in the upregulation of this gene [26]. SP1, which is a transcription factor with the binding site in the promoters of multiple genes, can induce the upregulation of TINCR [10], while miR-137/miR-133a directly targets TINCR and suppresses it [25]. In addition, it has been shown that the upregulation of TINCR is mainly caused by gene amplification in the genome [24]. A number of studies have reported high expression of TINCR in breast carcinoma, especially the HER2-positive subtype, while has been demonstrated that it is related to relapse-free survival (RFS), size of the tumor, and poor tumor prognosis in breast cancer [10, 24, 26, 27]. Moreover, TINCR negatively regulates apoptosis and triggers cell cycle progression [24]. Xu et al (2017) pointed out that the overexpression of TINCR could promote BC tumourigenesis through the TINCR-miR125b-HER2 axis [24, 26]. Dong et al. (2019) reported 
that overexpression of TINCR in HER2-positive BC patients was associated with poor response to trastuzumab therapy [26]. In vitro experiments have shown that TINCR reversed the resistance to trastuzumab and the acquired EMT in breast cancer cell lines. TINCR was detected in the cytoplasm, and it could sponge miR-125b in breast cancer cells, thereby releasing HER-2 and Snail-1 induce trastuzumab resistance [26]. Liu et al (2018) suggested that the TINCR-miR7KLF4 axis played a role in breast carcinoma. Indeed, TINCR, is a sponge for miR-7 released KLF4, which is a transcription factor with various physiological and pathophysiological functions [10]. Here, we detected the overexpression and hypomethylation of the TINCR gene in breast tumor tissues. Furthermore, we reported circulating level of TINCR was significantly higher in the plasma of patients, compared to healthy controls. The comparison of the c.TINCR sensitivity with CA15-3 revealed the potential diagnostic value of this lncRNA in plasma of patients with invasive ductal carcinoma of the breast. For the first time, we reported a lowfrequency rate of methylation in the promoter of the TINCR gene.

\section{Conclusions}

In addition to the overexpression of RP11-445H22.4 and TINCR genes, this is the first report to investigate the methylation of these two lncRNAs in breast tumors. Overall, it can be concluded that the expression levels and promoter methylation of RP11-445H22.4 and TINCR can be considered as potential biomarkers and therapeutic targets for breast carcinoma. Undeniably, more investigations on a larger population are required to validate the results of this pilot study. 


\begin{abstract}
Abbreviations
lncRNA: long non-coding RNA

c.KCNK15-AS1: circulating KCNK15-AS1

c.TINCR: circulating TINCR

ELISA: Enzyme-Linked Immunosorbent Assay

IDC: Invasive Ductal Carcinoma

TINCR: Tissue differentiation-Inducing long Non-Coding RNA
\end{abstract}

\title{
Data Availability
}

All relevant data are within the paper.

\section{Conflict of interest}

The authors declare no conflict of interest in this study.

\section{Authors' contributions}

M-T.M.; Performed all experiments, analyzed the data and wrote the initial draft of the manuscript. Z.R.; Contributed to concept and design, financial support, and revised the manuscript. All authors read and approved the final manuscript.

\section{Funding Statement}

This study has been funded by Kermanshah University of Medical Sciences, Iran [grant number: 95680, 2017]. 


\section{Acknowledgments}

We would like to thank all of the breast cancer patients who agreed to participate in this study. We also would like to appreciate all the staff at the Bistoon Hospital (Kermanshah, Iran) for their assistance in sample collection.

\section{ORCID}

Rahimi Z. (Corresponding author): http://orcid.org/0000-0001-7589-3307

Moradi, M-T. http://orcid.org/0000-0002-0247-5451 
Reference:

[1] Yari K., Rahimi Z., Payandeh M., and Rahimi Z. MMP-7 A-181G polymorphism in breast cancer patients from western Iran Breast Care, 2015. 10(6): p. 398-402.

[2] Moradi M.-T., Hatami R., and Rahimi Z. Circulating CYTOR as a Potential Biomarker in Breast Cancer International Journal of Molecular and Cellular Medicine (IJMCM), 2020. 9(1): p. 0-0.

[3] Jiang L.-h., Sun D.-W., Hou J.-C., and Ji Z.-I. CircRNA: a novel type of biomarker for cancer Breast cancer, 2018. 25(1): p. 1-7.

[4] Hanly D., Esteller M., and Berdasco M. Altered Long Non-coding RNA Expression in Cancer: Potential Biomarkers and Therapeutic Targets? 2019.

[5] Moradi M.-T., Rahimi Z., and Vaisi-Raygani A. New insight into the role of long non-coding RNAs in the pathogenesis of preeclampsia Hypertension in pregnancy, 2019. 38(1): p. 41-51.

[6] Yari K. and Rahimi Z. Promoter Methylation Status of the Retinoic Acid Receptor-Beta 2 Gene in Breast Cancer Patients: A Case Control Study and Systematic Review Breast Care, 2019. 14(2): p. 117-123.

[7] Wang X.-X., Guo G.-C., Qian X.-K., Dou D.-W., et al. miR-506 attenuates methylation of IncRNA MEG3 to inhibit migration and invasion of breast cancer cell lines via targeting SP1 and SP3 Cancer cell international, 2018. 18(1): p. 171.

[8] Moradi M.T., Fallahi H., and Rahimi Z. Interaction of long noncoding RNA MEG3 with miRNAs: A reciprocal regulation Journal of cellular biochemistry, 2019. 120(3): p. 3339-3352.

[9] Xu N., Chen F., Wang F., Lu X., et al. Clinical significance of high expression of circulating serum IncRNA RP11-445H22. 4 in breast cancer patients: a Chinese population-based study Tumor Biology, 2015. 36(10): p. 7659-7665.

[10] Liu Y., Du Y., Hu X., Zhao L., et al. Up-regulation of ceRNA TINCR by SP1 contributes to tumorigenesis in breast cancer BMC cancer, 2018. 18(1): p. 367.

[11] Kretz M., Siprashvili Z., Chu C., Webster D.E., et al. Control of somatic tissue differentiation by the long non-coding RNA TINCR Nature, 2013. 493(7431): p. 231.

[12] Moradi M.T., Yari K., and Khodarahmi R. A novel, efficient, fast and inexpensive DNA extraction protocol from whole blood applicable for studying drug-DNA interaction J Rep Pharm Sci, 2014. 3(1): p. 80-84.

[13] Shi T., Gao G., and Cao Y. Long noncoding RNAs as novel biomarkers have a promising future in cancer diagnostics Disease markers, 2016. 2016.

[14] Organization W.H. Biomarkers in risk assessment: Validity and validation 2001.

[15] Goossens N., Nakagawa S., Sun X., and Hoshida Y. Cancer biomarker discovery and validation Translational cancer research, 2015. 4(3): p. 256.

[16] Wang W., Xu X., Tian B., Wang Y., et al. The diagnostic value of serum tumor markers CEA, CA199, CA125, CA15-3, and TPS in metastatic breast cancer Clinica Chimica Acta, 2017. 470: p. 51-55.

[17] Chandra Gupta S. and Nandan Tripathi Y. Potential of long non-coding RNAs in cancer patients: from biomarkers to therapeutic targets International journal of cancer, 2017. 140(9): p. 19551967.

[18] Bussemakers M.J., Van Bokhoven A., Verhaegh G.W., Smit F.P., et al. Dd3:: A new prostatespecific gene, highly overexpressed in prostate cancer Cancer research, 1999. 59(23): p. 59755979.

[19] Groskopf J., Aubin S.M., Deras I.L., Blase A., et al. APTIMA PCA3 molecular urine test: development of a method to aid in the diagnosis of prostate cancer Clinical chemistry, 2006. 52(6): p. 1089-1095. 
[20] Jiao Z., Tian Q., Li N., Wang H., et al. Plasma long non-coding RNAs (IncRNAs) serve as potential biomarkers for predicting breast cancer Eur Rev Med Pharmacol Sci, 2018. 22(7): p. 1994-1999.

[21] He Y., Hu H., Wang Y., Yuan H., et al. ALKBH5 inhibits pancreatic Cancer motility by decreasing long non-coding RNA KCNK15-AS1 methylation Cellular Physiology and Biochemistry, 2018. 48(2): p. 838-846.

[22] Zhang H., Zhang Z., and Wang D. Epigenetic regulation of IncRNA KCNKI5-ASI in gastric cancer Cancer Management and Research, 2019. 11: p. 8589.

[23] Agrawal A., Murphy R.F., and Agrawal D.K. DNA methylation in breast and colorectal cancers Modern Pathology, 2007. 20(7): p. 711.

[24] Xu S., Kong D., Chen Q., Ping Y., et al. Oncogenic long noncoding RNA landscape in breast cancer Molecular cancer, 2017. 16(1): p. 129.

[25] Tian F., Xu J., Xue F., Guan E., et al. TINCR expression is associated with unfavorable prognosis in patients with hepatocellular carcinoma Bioscience reports, 2017. 37(4): p. BSR20170301.

[26] Dong H., Hu J., Zou K., Ye M., et al. Activation of LncRNA TINCR by H3K27 acetylation promotes Trastuzumab resistance and epithelial-mesenchymal transition by targeting MicroRNA-125b in breast Cancer Molecular cancer, 2019. 18(1): p. 3.

[27] Li J., Gao C., Liu C., Zhou C., et al. Four IncRNAs associated with breast cancer prognosis identified by coexpression network analysis Journal of cellular physiology, 2019. 234(8): p. 14019-14030. 
Table 1. The sequence of primers and PCR product size for qRT- PCR and MSP.

\begin{tabular}{|c|c|c|c|}
\hline Assay & Sequence of forward primer $\left(5^{\prime}->3^{\prime}\right)$ & Sequence of reverse primer $\left(5^{\prime}->3^{\prime}\right)$ & Product size \\
\hline \multicolumn{4}{|l|}{ qRT-PCR $^{1}$} \\
\hline KCNK15-AS1 & gtaaagccatcaccaggacaacc & ctccctaacagaagcccacca & 159-bp \\
\hline TINCR & tgtggcccaaactcagggatacat & agatgacagtggctggagttgtca & 213-bp \\
\hline U6 snRNA & ctcgcttcggcagcaca & aacgcttcacgaatttgcgt & 94-bp \\
\hline \multicolumn{4}{|l|}{$\mathbf{M S P}^{2}$} \\
\hline $\mathrm{M}^{3} \cdot \mathrm{KCNK} 15-\mathrm{AS} 1$ & atagatatatgaatttcgggtaggc & attataacgaacgaaacgaaacg & 123-bp \\
\hline $\mathrm{U}^{4} \cdot \mathrm{KCNK} 15-\mathrm{AS} 1$ & agatatatgaattttgggtaggtga & attataacaaacaaaacaaaacaac & 121-bp \\
\hline M.TINCR & ggttagagttggagtcggag & ggggttagagttggagttgg & 90-bp \\
\hline U.TINCR & atacaccttaatataataacgcttccaac & ccaaatacaccttaatataataacactt & 96-bp \\
\hline
\end{tabular}


Table 2. Some clinicopathological characteristics of the patients.

\begin{tabular}{cc}
\hline Variable & Patients (\%) \\
\hline $\begin{array}{c}\text { Histological type: } \\
\text { Invasive ductal carcinoma }\end{array}$ & $40(100)$ \\
\hline Tumor Stage & $11(27.5)$ \\
I & $19(47.5)$ \\
II & $10(25)$ \\
III & \\
ER & $9(32.5)$ \\
Negative & $31(77.5)$ \\
Positive & $12(30)$ \\
PR & $28(70)$ \\
Negative & \\
Positive & $21(52.5)$ \\
HER2 & $19(47.5)$ \\
Negative & \\
Positive & $0(0)$ \\
Ki67 & $40(100)$ \\
Negative &
\end{tabular}


Table 3. Correlation between promoter methylation and gene expression in the three lncRNAs.

\begin{tabular}{|c|c|c|c|c|}
\hline \multirow[b]{2}{*}{ Promoter status } & \multirow[b]{2}{*}{$\mathbf{N}(\%)$} & \multicolumn{3}{|c|}{ One way ONNOVA } \\
\hline & & Ave. of $\Delta_{\mathrm{Ct}}$ & F, R square & $P$-value \\
\hline \multicolumn{5}{|l|}{ KCNK15-AS1 } \\
\hline Fully methylated & $12(30)$ & 4.25 & & \\
\hline Hemi-methylated & $10(25)$ & 3.36 & $15.72,0.46$ & $<0 / 0001$ \\
\hline Un-methylated & $18(45)$ & 2.63 & & \\
\hline \multicolumn{5}{|c|}{ Pearson correlation: $\mathrm{r}=-0.68,95 \% \mathrm{CI}(-0 / 82$ to $-0 / 46), \mathrm{P}<0 / 0001$} \\
\hline \multicolumn{5}{|l|}{ TINCR } \\
\hline Fully methylated & $15(37.5)$ & 4.19 & & \\
\hline Hemi-methylated & $9(22.5)$ & 2.51 & $54.84,0.75$ & $<0 / 0001$ \\
\hline Un-methylated & $16(40)$ & 1.87 & & \\
\hline \multicolumn{5}{|c|}{ Pearson correlation: $\mathrm{r}=-0.84,95 \% \mathrm{CI}(-0 / 91$ to $-0 / 72), \mathrm{P}<0 / 0001$} \\
\hline
\end{tabular}


Relative expression of the lncRNAs in tumor tissues

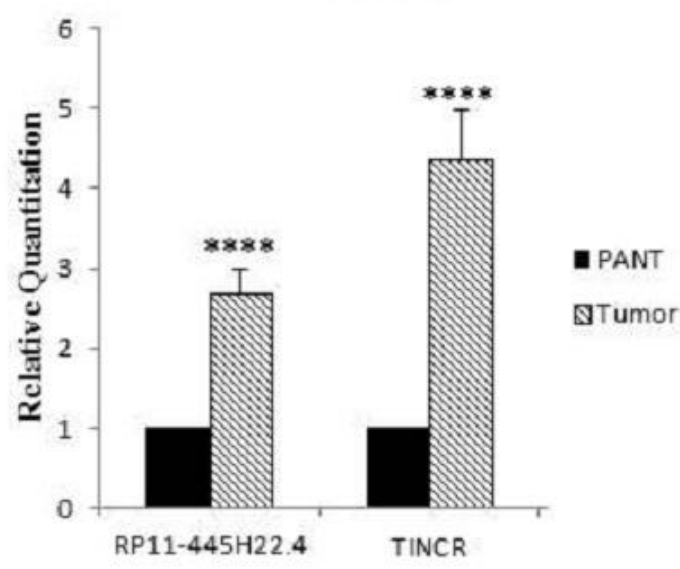

(a)
Relative levels of the circulating lncRNAs

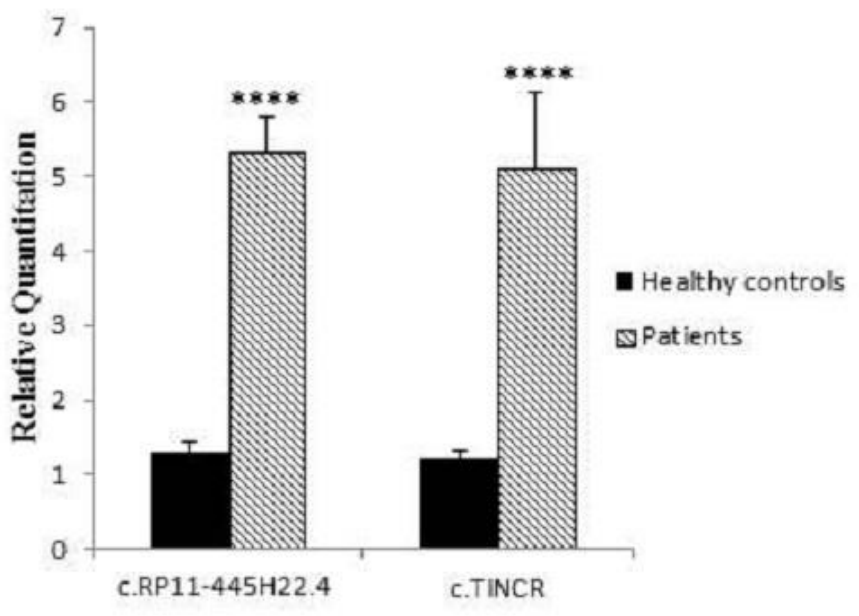

(b)

Figure 1. (a) Relative expression of RP11-445H22.4 and TINCR in paired breast tumor and adjacent non-tumoral tissues; (b) relative expression levels of c.RP11-445H22.4 and c.TINCR in plasma of breast cancer patients in comparison with the plasma of healthy controls. 


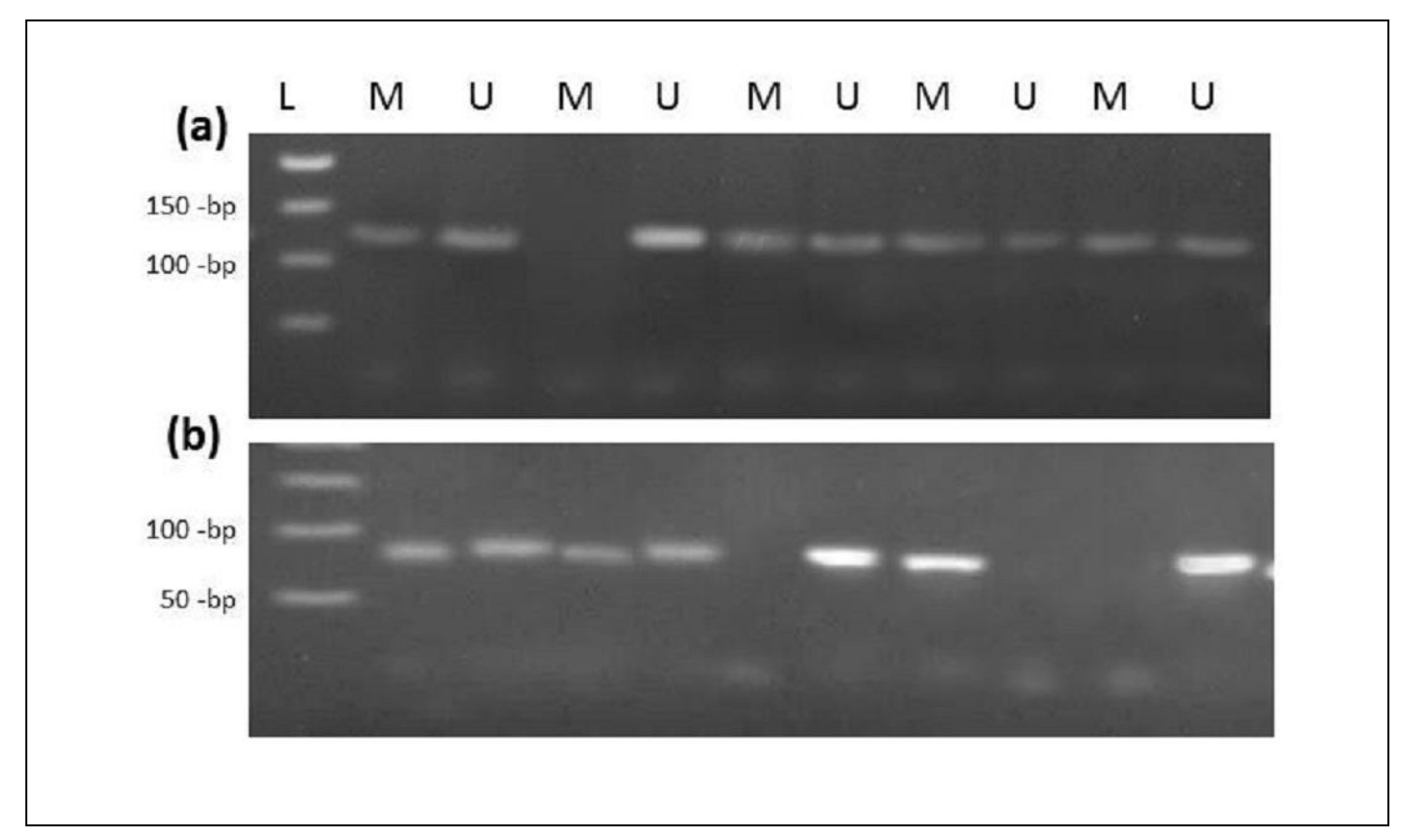

Figure 2. Agarose gel electrophoresis of MSP products of (a) RP11-445H22.4 and (b) TINCR genes. The first lane (L) shows a 50-bp DNA ladder, and each other lanes show methylated (M) and unmethylated (U) PCR products for each sample. 


\section{a}

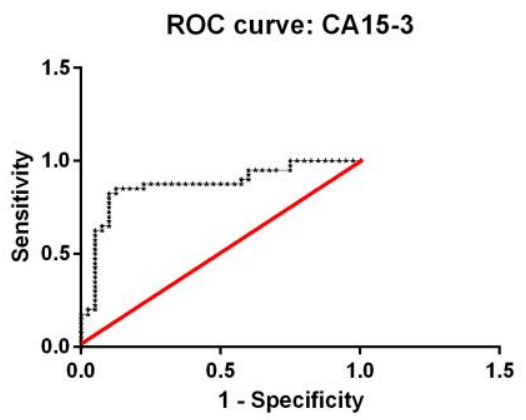

b

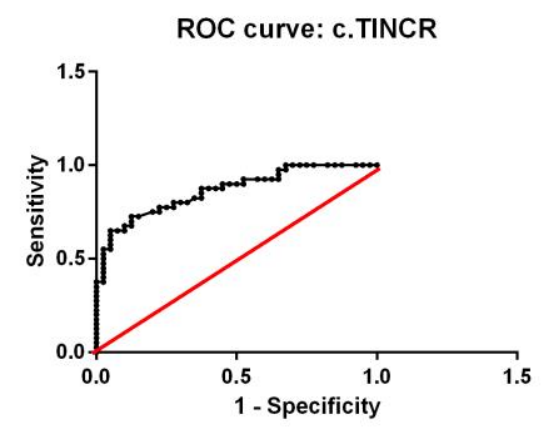

C

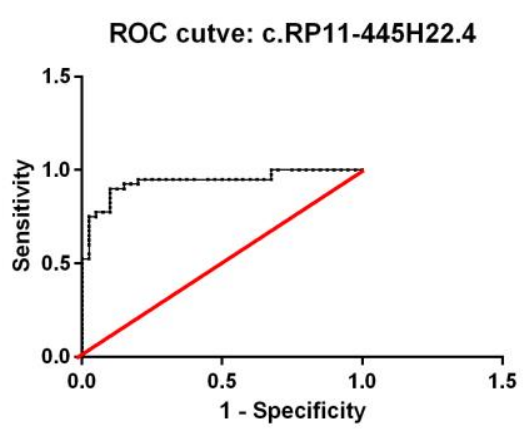

Figure 3. ROC curve for evaluation of the diagnostic value of CA 15-3 (a) circulating TINCR (b), and circulating RP11-445H22.4 (c) in plasma of breast cancer patients and healthy control donors. 


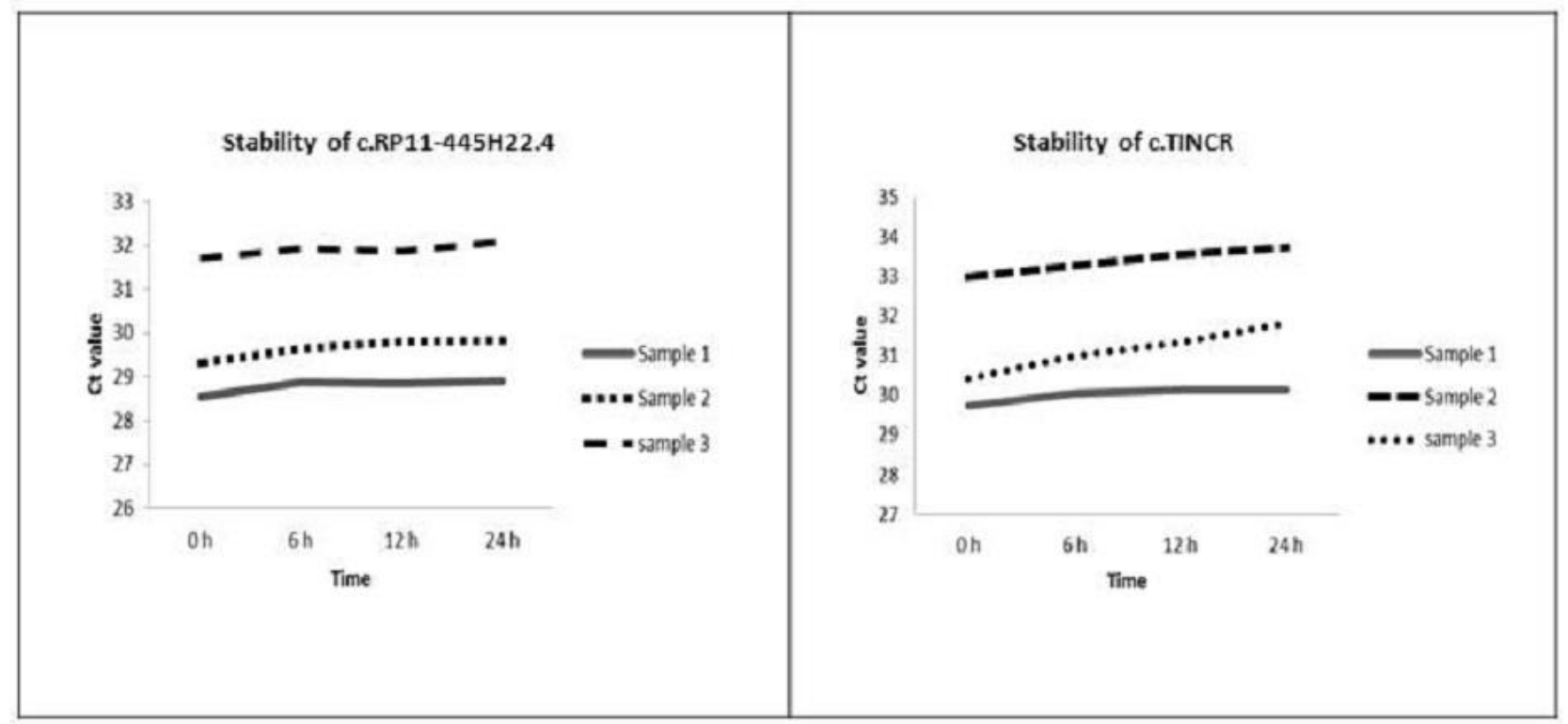

Figure 4. The stability assessment of c.RP11-445H22.4 and c.TINCR of three samples right after sampling (0h), and also after 6-, 12-, and 24-hour incubation of plasma samples at room temperature. 